\title{
Ossifying Fibroma of Nasal Cavity: A Rare Case Report
}

\author{
Tulachan Bishow* \\ Department of ENT-Head and Neck Surgery, Universal College of Medical Sciences, Nepal
}

*Corresponding author: Bishow Tulachan, Department of ENT-Head and Neck Surgery, Universal College of Medical Sciences, Tribhuvan University Teaching Hospital, Nepal.

Received Date: November 05, 2018

Published Date: December 11, 2018

\begin{abstract}
Ossifying fibroma is a rare benign fibro-osseous lesion more commonly seen in the head and neck regions. Despite a benign lesion, it behaves aggressively locally and possess higher recurrence nature. One may have a diagnostic challenge owing to its rapid progression and osteolytic nature and having the impression of malignant lesion. Here, we are presenting a case of 9 years old girl with complaints of left nasal obstruction and left facial swelling for 6 months.
\end{abstract}

Key words: Fibroma; Osteolytic; Recurrence

\section{Introduction}

Ossifying fibroma (OF) is a rare slow-growing benign tumor of fibro osseous tissue with characteristics of aggressive nature locally and mimicking malignant lesion [1].

Menzel initially described it in 1872 and later the term was coined by Montgomery in 1927 and are commonly found in the head and neck regions. These tumors are commonly cited in the mandible (62-89\%), maxilla, parietal, temporal, sphenoid bones, nasopharynx, sella turcica, orbit, skull base, calvarium and nasal cavity. The Sino nasal tract is one of the rarest sites for its occurrence [1-3].

The cause of OF is unknown however several hypothetical theories like odontogenic, developmental and traumatic have been suggested. The study has shown female predilection over male with ratio of 2:1:3. Hereditary predominance has not been demonstrated as per the studies [2]. First and second decade age groups show high predilection for head and neck OF [4].

Sino nasal tract OF typically demonstrates aggressive pattern locally with rapid growth however the mandibular lesions do present as painless slow growing tumors. Here, we are reporting a rare case of ossifying fibroma in the left nasal cavity with facial asymmetry and left proptosis.

\section{Case report}

A 9 years old girl was referred to the otolaryngology clinic from the Eye hospital with 6 months history of nasal obstruction, nasal deformity, left periorbital swelling and headache. Examination revealed broadened nasal dorsum, left orbital swelling, grossly deviated nasal septum to the right with left facial asymmetry (Figure 1A). The left nasal cavity was completely occupied by a soft to firm mass (Figure 1B). Her vision was within normal limit as per the Eye hospital reports. Oral cavity and oropharynx were normal. Posterior rhinoscopy couldn't be done. CT (computerized tomography) of nose and paranasal sinuses had impression of irregular expansile hyperdense lesion involving left nasal cavity, ipsilateral maxillary and ethmoid sinuses with cortical thinning and focal cortical break of lateral and anterior maxillary sinus walls and mild bone remodeling of the medial wall of left orbit and deviated nasal septum towards right without intracranial extension (Figure 2A). Also, a no enhancing hyperdense foci in right frontal lobe without surrounding perilesional edema or mass effect. Under general anesthesia, the mass was excised in toto (piecemeal) via lateral rhinotomy along with endoscopic approach however the exact size couldn't be measured (Figure 1B-1E). The histopathology report was suggestive of ossifying fibroma. The patient was symptom free on her post-operative days (Figure 1F). 


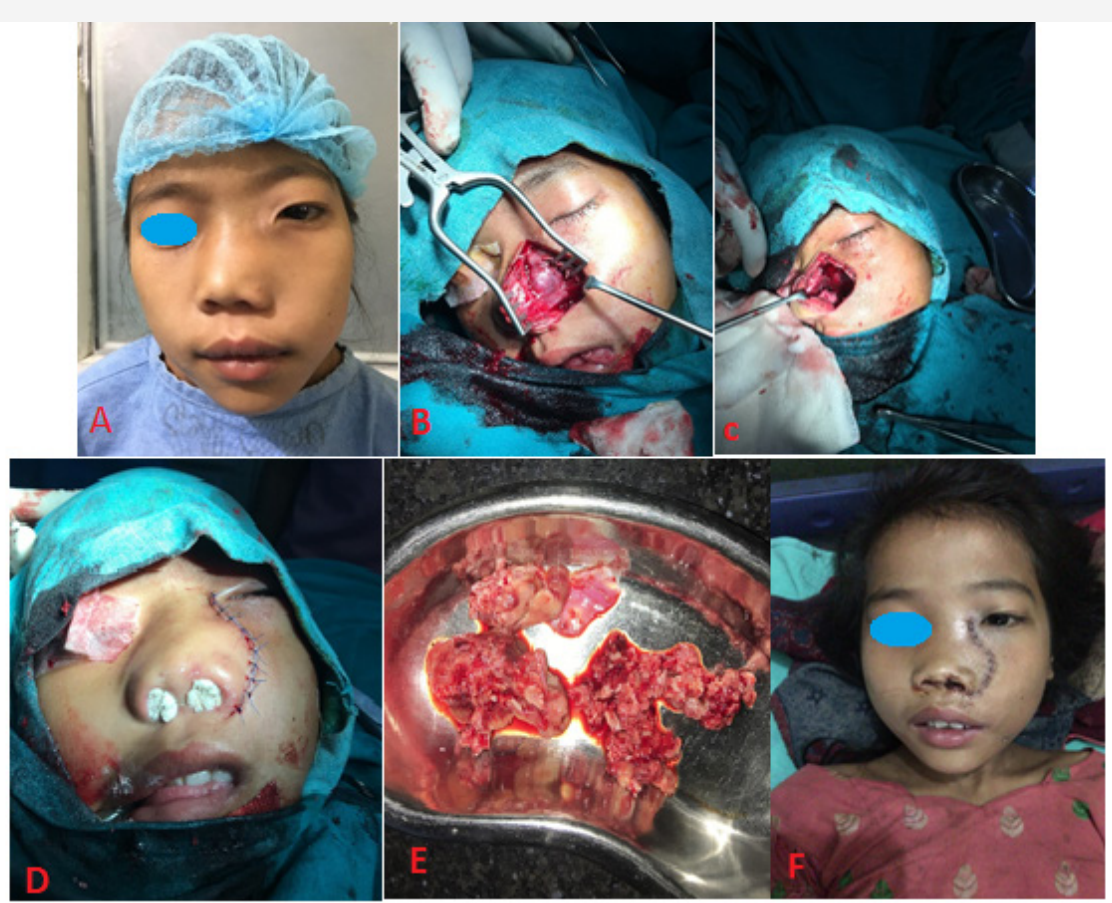

Figure 1: A- preoperative image, B- C-exposure of the mass via lateral rhinotomy, C- after the resection of the lesion, E- anterior nasal pack with the external sutures, D- the excised mass in toto, E- 7th post-operative day following pack and suture removal.
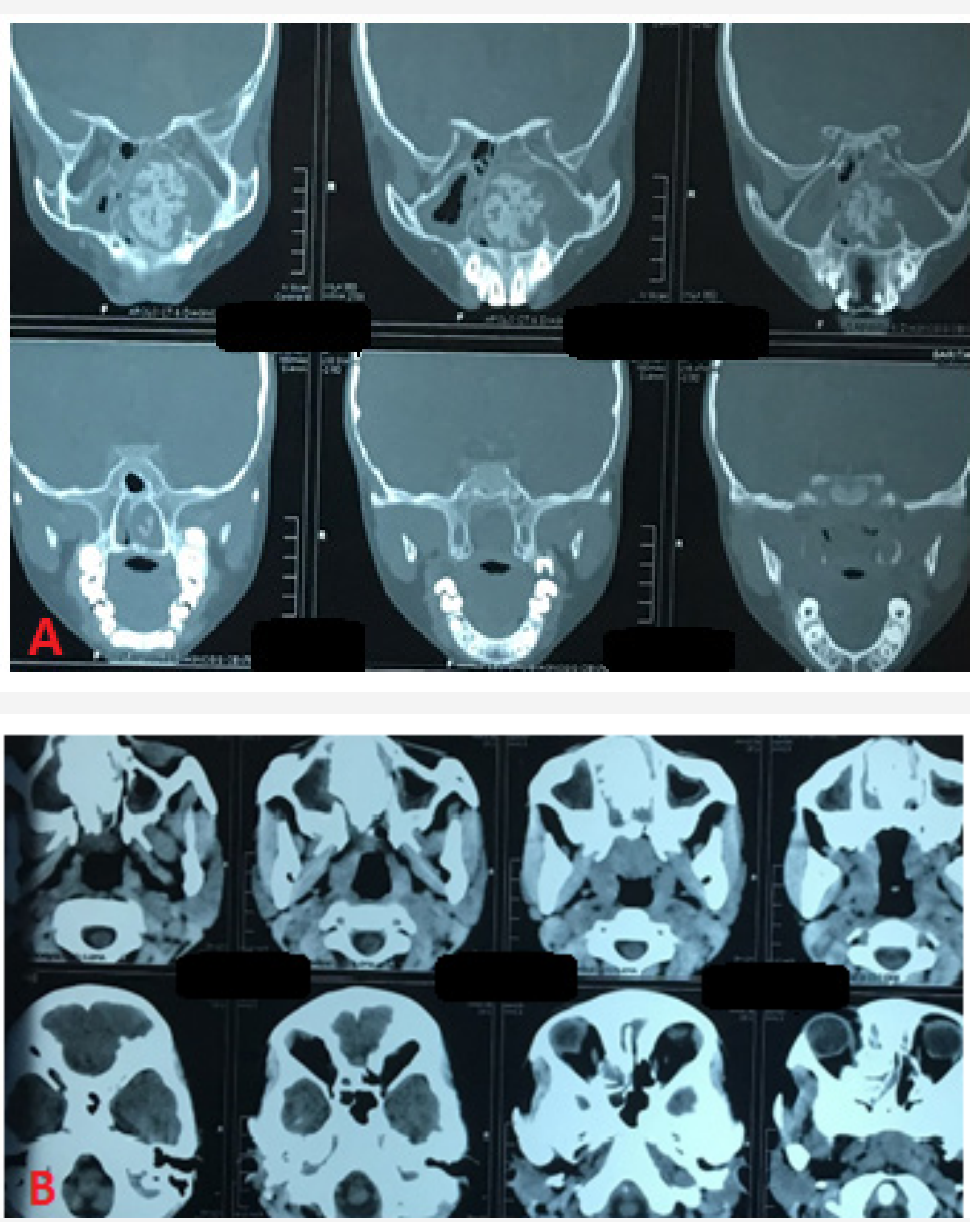

Figure 2: A\&B coronal and axial view of CT Paranasal sinuses

\section{Discussion}

There's a similarity among all the fibro osseous lesions i.e. the replacement of normal bone by a tissue full of collagen fibers and fibroblasts containing varying amounts of mineralized substance, which may be bony or cementum like or mixture of both [3]. Such lesions may contain trabecular of lamellar bone rimmed 
by osteoblasts with numerous small rounded psammoma like bodies present in the cellular tissue in $60 \%$ of cases [1,6]. WHO (World Health Organization) new classification has a new term as JOF (Juvenile Ossifying Fibroma) to those fast-growing mass between 5-15 years of age with well bordered on radiology with consistency of ossifying fibroma on histology $[7,8]$. JOF has two histological variants: Juvenile psammomata ossifying fibroma and juvenile trabecular. The psammomata type is more common than the trabecular variety, is more aggressive, and possess high recurrence tendency. It has characteristics of a well-defined, unilocular or multilocular expansile and well-circumscribed radiolucent lesions with cortical thinning radiologically [7-9]. It may invade the eye socket, skull base and calvarium and may present with related symptoms, and its malignancy rate is 0.4 $0.5 \%$ ). As per the studies, it seems to induce by repetitive surgery or chemotherapy [11]. Regarding the age, rapid progression of the lesion and the radiographic features, the case bears similarity with JOF. The closest condition that needs to be differentiated is from fibrous dysplasia and the differentiating features being fast growing lesion, monostatic nature and the well delineated radiographic margins [10]. Other differential diagnosis includes osteoblastoma, osteosarcoma, eventifying fibroma, and aneurysmal bone cyst [7]. The only effective treatment for such lesion is the complete resection [4] and there are many approaches for the access. Although the prognosis following complete resection is good, recurrence mostly occurs due to incomplete surgery. Patient needs to undergo redo surgery on recurrence. Radiotherapy and chemotherapy are not advised as it may transform the lesion into malignancy like osteosarcoma [11].

\section{Conclusion}

Although the lesion behaves aggressively locally, it can be diagnosed early with the clinical and radiological features in OPD (outpatient department) basis. The chance of recurrence is almost nil on complete surgical excision of the lesion.

\section{Acknowledgement}

None.

\section{Conflict of Interest}

There is no conflict of interest.

\section{References}

1. Zafar A, Shafi M, Malik S, Perviaz S (1197) Ossifying Fibroma of Nasal Cavity. Journal of Pakistan Medical Association 47(12): 312-313.

2. Pathak SN, Dubey A, Dubey A (2016) Case Report: A Rare Case of Ossifying Fibroma of the Nasal Cavity. Imperial Journal of Interdisciplinary Research 2(11): 743-744.

3. Khanna JN, Andrade NN (1992) Giant ossifying fibroma. Case report on a bimaxillary presentation. Int J Oral Maxillofac Surg. 21(4): 233-235.

4. Kumar S, Dar NH, Ashraf F, S C Sharma, V Maheshwari (2004) Ossifying fibroma of nose in a two-year-old child. Indian Journal of Otolaryngology and Head and Neck Surgery 56(1): 37-39.

5. Rahmat K, CH T, Kumar G (2007) Cemento ossifying fibroma of the paranasal sinuses: A review of two cases. Eur J Radiolog Extra 63(3): 101-104.

6. Bertrand B, Buoy PH, Comelis PH, Gosseye S, Clotuche J, et al. (1993) Juvenile aggressive cemento-ossifying aroma: Case report and review of the literature. Laryngoscope 103(12): 1385-1390.

7. Kundu PR, Beniwal K, Singh B, Kaur S (2014) Juvenile ossifying fibroma: An unusual case report and review of literature. Saudi Surg J 2: 57-59.

8. Keles B, Duran M, Uyar Y, Azimov A, Demirkan A, et al. (2010) Juvenile ossifying fibroma of the mandible: A case report. J Oral Maxillofac Res 1(2): 1-7.

9. Mehta D, Clifton N, McClelland L, Jones NS (2006) Paediatric fibroosseous lesions of the nose and paranasal sinuses. Int J Pediatr Otorhinolaryngol 70(2): 193-199.

10. Wenig BM, Vinh TN, Smirniotopoulos JG, Fowler CB, Houston GD, et al. (1995) Aggressive psammomatoid ossifying fibromas of the sinonasal region: A clinicopathologic study of a distinct group of fi bro-osseous lesions. Cancer 76(7): 1155-1165.

11. Sun N, Xu WH, Cao LH, Xiao Yan Zhao, Jing Fei Zhang, et al. (2013) A case of ossifying fibroma of the frontal sinus. Experimental and therapeutic science 5(5): 1359-1362. 